\title{
Characterization of poly(vinyl alcohol)- polycaprolactone hybridized scaffold for potential skin tissue regeneration
}

\author{
Tan Yong Chee ${ }^{a}$, Abdull Rahim Mohd Yusoff ${ }^{a}{ }^{*}$, Nik Ahmad Nizam Nik Malek ${ }^{b}$ \\ a Department of Chemistry, Faculty of Science, Universiti Teknologi Malaysia, 81310 Johor Bahru, Johor, Malaysia. \\ ${ }^{b}$ Department of Biosciences, Faculty of Science, Universiti Teknologi Malaysia, 81310 Johor Bahru, Johor, Malaysia. \\ * Corresponding author: rahim@kimia.fs.utm.my
}

\section{Article history}

Received 24 January 2019

Revised 7 Mac 2019

Accepted 15 October 2019

Publishe Online 2 February 2020

\begin{abstract}
The fabrication of a hybridized scaffold constituting hydrophobic and hydrophilic polymers for tissue engineering has received an increasing attention recently. Due to the high compatibility with water, a hydrophilic polymer, though is able to enhance cell affinity and proliferation, has a very high biodegradable rate and low stability in aqueous medium that eventually puncture its biomedical applications. Thereby, the addition of a hydrophobic polymer in the hydrophilic polymer scaffold is recommended to increase the hydrophobic property of the scaffold in order to reduce the limitation. Nonetheless, the fabrication of the hybridized scaffold is extremely challenging because the hydrophilic and the hydrophobic polymer tends to dissolve in different types of solvents, i.e. water and organic solvent, respectively, that subsequently restricts their blending process. In this work, a poly(vinyl alcohol) (PVA) scaffold, a polycaprolactone (PCL) scaffold, and their hybridized scaffold were produced through casting method for potential skin tissue regeneration. We found that the glacial acetic acid was an appropriate solvent used to prepare hydrophobic PCL solution with low molecular weight (16 kDa) for PCL-PVA blend, with mass ratio 1:1, without using any surfactant. The solvent was also used for the preparation of PCL scaffold with high molecular weight (80 kDa). The fabricated polymer scaffolds were then evaluated using FTIR-ATR, contact angle measurement, and tensile strength analysis. FESEM images of the PVA-PCL hybridized scaffold showed that the PCL was well dispersed in the PVA scaffold. FTIR-ATR spectra showed that the hybridized scaffold exhibited the crucial functional group of PVA and PCL at 3310.97, 1720.10, 1557.80, 1241.69, $1172.90,1044.95$, and $719.44 \mathrm{~cm}^{-1}$. The contact angle of the PVA, PCL, and PVA-PCL hybridized scaffold were $61.3^{\circ}, 82.7^{\circ}$, and $75.9^{\circ}$, respectively, with tensile strength $16.5747,2.4038$, and 7.417 $\mathrm{MPa}$, respectively.
\end{abstract}

Keywords: Poly(vinyl alcohol), polycaprolactone, casting, scaffold

(C) 2020 Penerbit UTM Press. All rights reserved

\section{INTRODUCTION}

Burn, one of the worst forms of trauma, is accounted for an increased worldwide incidence of approximately two million cases annually (Brigham and McLoughlin, 1996). To date, there are many methods have been introduced to treat varying degree of burn wound such as wound dressing, tissue culture, and skin grafting. A wound dressing is a type of skin repair therapy that has been widely used to treat minor skin injury. It is not suitable for patients with extensive skin loss because it is a time-consuming process (Bloemen et al., 2012; Ulrich et al., 2014). Tissue culture is another method that involves culturing a specific type of cell tissue in a suitable medium prior to the treatment of burn wound. Although this method can be used to cure patients with a series burn wound, it posses some drawbacks such as slow cell tissue culturing and poor cell tissue growing (Chio and Agrawal, 2010). Skin grafting is a type of graft surgery involving the transplantation of skin tissue. This treatment is effective, but it may result in disease transmission and post-surgical adhesion. Post-surgical adhesion is a problem that requires patients have to go through a second surgery after grafting, and this subsequently induces scar tissue formation and infection. Furthermore, the supply of grating of tissue is very limited (Kumbar et al., 2008). Thereby, using polymer to produce skin scaffold are recommended worldwide.

In most cases, hydrophilic polymer tends to be used as a promising candidate for scaffold formation. This is because this kind of polymer has $\mathrm{OH}$ - bond that can improve the cells attachment and proliferation and eventually enhance the wound healing process. However, due to the hydrophilic property, this kind of polymer has a low stability when contacting with water. The hydrogen bonding between the water and the scaffold causes the scaffold to dissolve in water very quickly. Thereby, hydrophobic polymer was proposed to solve the problem (Hiep and Lee, 2010). Unlike hydrophilic polymer, the hydrophobic polymer has a relatively strong stability when in contact with water. Nevertheless, the hydrophobic polymers are not suitable for biomedical application because of the lack of $\mathrm{OH}$ - bond that hinders the cell attachment and proliferation (Cossio et al., 2012).

Poly(vinyl alcohol) (PVA) is a highly hydrophilic synthetic polymer. It has been widely employed in biomedical application because of its excellent physicochemical properties (Shalumon et al., 
2009; Park et al., 2010) such as non-toxic, biodegradable, biocompatible, and has a high ability to form hydrogels, fibers, and films (Shao et al., 2003). To date, PVA has been used in the production of contact lenses, artificial organs, and drug delivery systems (Zhang et al., 2005). On the other hand, polycaprolactone (PCL) is a hydrophobic polymer with low melting point. Due to its flexible chains at room temperature, the PCL can be dissolved in many organic solvents such as acetone, chloroform, dichloroethane, dimethylformamide, hexafluoroisopropanol, methylene chloride, tetrafluoroethylene, and pyridine (Cossio et al., 2012; Ferreira et al., 2014) and has a good miscibility with other polymer (Woodruff and Hutmacher, 2010). Recently, PCL scaffolds have been used as an artificial bone tissue. The product exhibits a high compatibility with a wide range of pharmaceutical agents in which enable the drug to be distributed uniformly in a formulation. Additionally, its long-term degradation makes it a suitable material for controlled delivery applications up to several months (Yoshimoto et al., 2003).

In this study, we proposed a combination of a hydrophilic polymer, i.e. PVA, and a hydrophobic polymer, i.e. PCL, to form a hybridized PVA-PCL film via casting method for potential skin tissue regeneration. Both polymers have been approved by the U.S. Food and Drug Administration (FDA) (Woodruff and Hutmacher, 2010; Zhang et al., 2005). The combination of PVA and PCL was very challenging because they tend to reject each other when their solutions are mixed. Characterization methods, namely field emission scanning electron microscopy (FESEM), Fourier-transform infra-red spectroscopy (FTIR), contact angle measurement, and tensile strength test were also conducted to investigate the physicochemical properties of the hybridized film toward potential skin tissue regeneration.

\section{EXPERIMENTAL}

\section{Materials}

Polycaprolactone with different molecular weights (PCL, Mw = $16,000 \mathrm{~g} / \mathrm{mol} ; \mathrm{PCL}^{*}, \mathrm{Mw}=80,000 \mathrm{~g} / \mathrm{mol}$ ) were obtained from Aldrich Chemistry and used without further purification. Poly(vinyl alcohol) (PVA, Mw = 15,000 g/mol) was obtained from Merck KGaA and used without further purification Glacial acetic acid was purchased from HmbG Chemical and used without any purification. The ultrapure water was obtained from Arium-Pro ultrapure water system.

\section{Preparation of PVA, PCL*, and PVA-PCL film}

Fig. 1 illustrates the preparation of polymer film via casting method. The polymer solution was poured on a glass plate and moulded into a flat rectangular sheet using a glass rod. After that, the polymer solution was dried at ambient condition (air dry) until a film was formed. For PVA solution, $2.0 \mathrm{~g}$ of PVA was dissolved in $10 \mathrm{~mL}$ of ultrapure water and stirred at $100{ }^{\circ} \mathrm{C}$ until the solution was homogenized. For PCL $(16,000$ and $80,000 \mathrm{~g} / \mathrm{mol})$ solutions, $2.0 \mathrm{~g}$ of PCL and PCL* were dissolved in glacial acetic acid, separately, and stirred at room temperature until homogenous. For hybridized PVAPCL solution, the PVA solution and the PCL solution were blended in a ratio of $1: 1$ and stirred at $60^{\circ} \mathrm{C}$ for $5 \mathrm{~h}$.

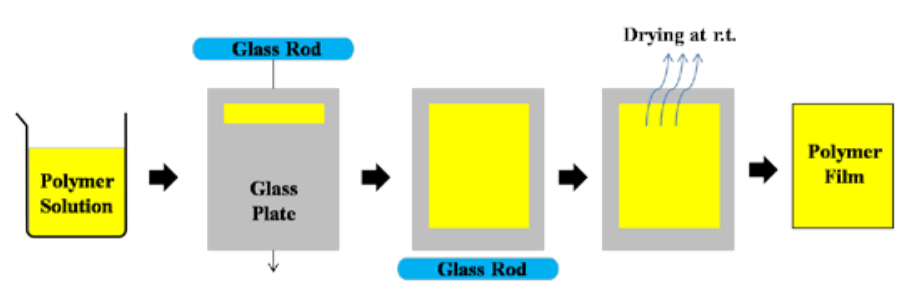

Fig. 1 Schematic diagram for preparation of polymer film via casting method.

\section{Field Emission Scanning Electron Microscope}

The surface morphology of the PVA-PCL film was observed using FESEM (Zeiss Supra 35VP) operated at $5 \mathrm{kV}$. The function of FESEM was to observe the surface morphology and size of bulk single crystals formed in the polymer film. Before the observations,

all samples were sputter-coated with gold at $10^{-1}$ Mbar using Bio Rad Polaron Division SEM sputter coater.

\section{Fourier-transform infra-red spectroscopy}

The study of functional groups on the surface of membrane was carried out by using PerkinElmer Frontier FTIR spectrometer equipped with ATR. The ATR mode was chosen in this study because it is a non-disruption analysis and allows for point-to-point examination.

\section{Contact angle measurement}

The static surface contact angle of the polymer film was measured using contact angle analysis system from physics OCA. A few droplets of distilled water were dispensed onto the fiber surface by using a sessile drop method. A picture of the droplet is captured after the droplet sat onto the membrane surface. Then, the angle of the droplets on the surface of the membrane was measured and recorded by the system. The contact angle for each membrane is obtained from an average of 5 measurements of each membrane. Finally, the relative hydrophilicity of the membrane was qualitatively determined by referring to the contact angle.

\section{Tensile strength tester}

The mechanical properties of the polymer film were measured by LRX $2.5 \mathrm{kN}$ Lloyd tensile tester. Before the testing, the area and the thickness of each polymer film were measured because both of the measurements are the key factors used in measuring the tensile strength. The area of each membrane was fixed at dimension $30 \mathrm{~mm} \mathrm{x}$ $13 \mathrm{~mm}$ and their thickness was also measured using electronic external micrometer. The controlled force module was selected with speed set at $10 \mathrm{~mm} / \mathrm{min}$. In order to obtain a more accurate result, each measurement was repeated 5 times and their mean is taken as their tensile strength.

\section{RESULTS AND DISCUSSION}

Preparation of PVA, PCL, and PVA-PCL* hybridized film

Fig. 2 illustrates the condition of each polymer solution. It was noted that the glacial acetic acid was a good solvent for PCL, regardless of molecular weight. Additionally, the usage of acetic acid is highly recommended because the acid is less harmful for human (up to $20 \%$ in vinegar) and have been commonly used in wound healing applications. For instance, it has been directly applied on infected burns and wounds, in the form of $1-5 \%(\mathrm{v} / \mathrm{v})$ solution in water, to inhibit the growth of bacteria such as Escherichia coli, Staphylococcus aureus, and Pseudomonas aeruginosa. (Contardi et al., 2017). Thereby, the production of scaffold using acetic acid can prevent bacteria colonization during the process.

When PVA solution and PCL solution were blended, a white emulsion was formed as shown in Fig. 2(d), without using any surfactant or emulsifier. The formation similar as the water-oil emulsion. The white emulsion of PVA-PCL was considered very stable because it can remain the emulsion form up to several days with continuous stirring at $60^{\circ} \mathrm{C}$.
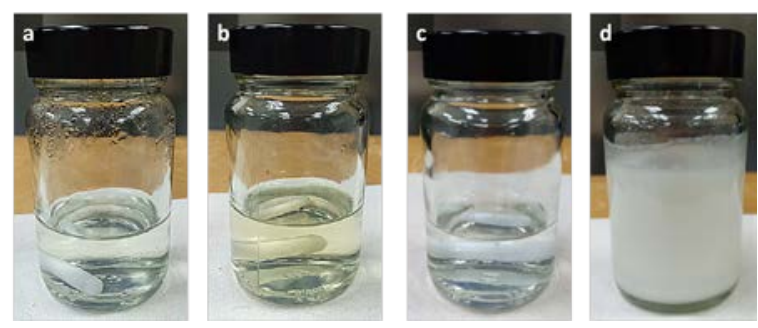

Fig. 2 solution.

After casting, all polymer solutions formed films, except the PCL solution. As a matter of fact, the film formation of a polymer is related to the intermolecular forces between the polymers in a solution. Owing to the polymer chain of PVA consists of $\mathrm{OH}$ - bond, they create 
a very strong hydrogen bonding among the PVA. Thus, the PVA tends to attract each other and form film easily even though in a low molecular weigh form. In the case of PCL, the intermolecular forces between them is merely Van der Waals forces. Therefore, only the high molecular weight PCL* has enough intermolecular forces to aggregate and form film. Low molecular weight PCL tends to form powder after casting as shown in Fig. 3 (c). Fig. 3 shows the condition of polymer films after casting. PVA solution produced a transparent film, PCL* solution produced a white film, while the PVA-PCL solution produced film consisted of white spot and transparent areas that are arranged uniformly. This phenomenon indicated that the PVA and PCL were dispersed evenly when they were in emulsion and film as the glacial acetic acid and water were miscible to each other. FESEM images of PVA-PCL hybridized film (Fig. 4) also prove that the PVA and PCL dispersed evenly throughout the hybridized film. PCL was chosen to blend with PVA rather than PCL* because the high molecular weight PCL* formed white solid when contact with PVA solution.
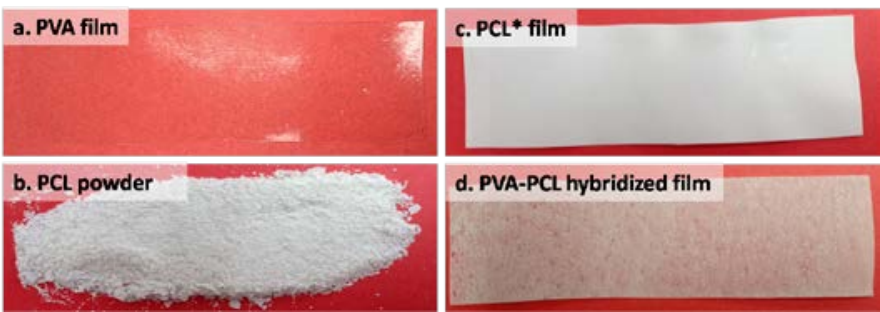

d. PVA-PCL hybridized film

Fig. 3 (a) PVA film, (b) PCL powder, (c) PCL* film and (d) PVA-PCL hybridized film.

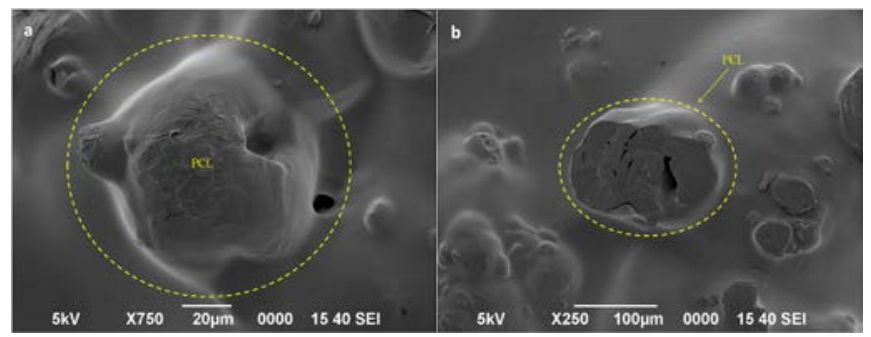

Fig. 4 FESEM images of PVA-PCL film at magnifications of (a) 750 and (b) 250 times

In short, the production of PVA-PCL hybridized film using water and glacial acetic acid was a promising method because not only it produced film with well-dispersed PVA and PCL, but can also prevent bacteria colonization during the production.

\section{Physicochemical properties of PVA, PCL*, and PVA-PCL hybridized film}

Fig. 5 illustrates the FTIR-ATR spectra of PVA film, PCL* film, and PVA-PCL hybridized film. Results show that the PVA-PCL hybridized film contained the inherent functional group of PVA (green line) and PCL (red line) after casting. FTIR-ATR spectra show that the hybridized scaffold exhibited the crucial functional group of PVA and PCL at 3285.37, 1712.02, 1566.46, 1360.92, 1284.12, and $719.44 \mathrm{~cm}^{-1}$ corresponding to the $\mathrm{O}-\mathrm{H}$ stretching (for PVA), $\mathrm{C}=\mathrm{O}$ stretching (for PCL), O-H bending vibrations (for PVA), C-O-C asymmetric stretching (for PCL), C-O-C stretching (for PCL), and $\mathrm{C}=\mathrm{O}$ stretching (for PCL) (Acess et al., 2016; Liu et al., 2017; Santos et al., 2014; Wang 2012). This result indicates that the combination of PVA and PCL via the proposed method can combine the PVA and PCL effectively into a single film without losing any important functional group of PVA and PCL. In other word, the proposed method successfully increased hydrophilic property of PCL film by combining the hydrophilic functional group of PVA, that subsequently can improve the interaction between the cell and the designed scaffold.

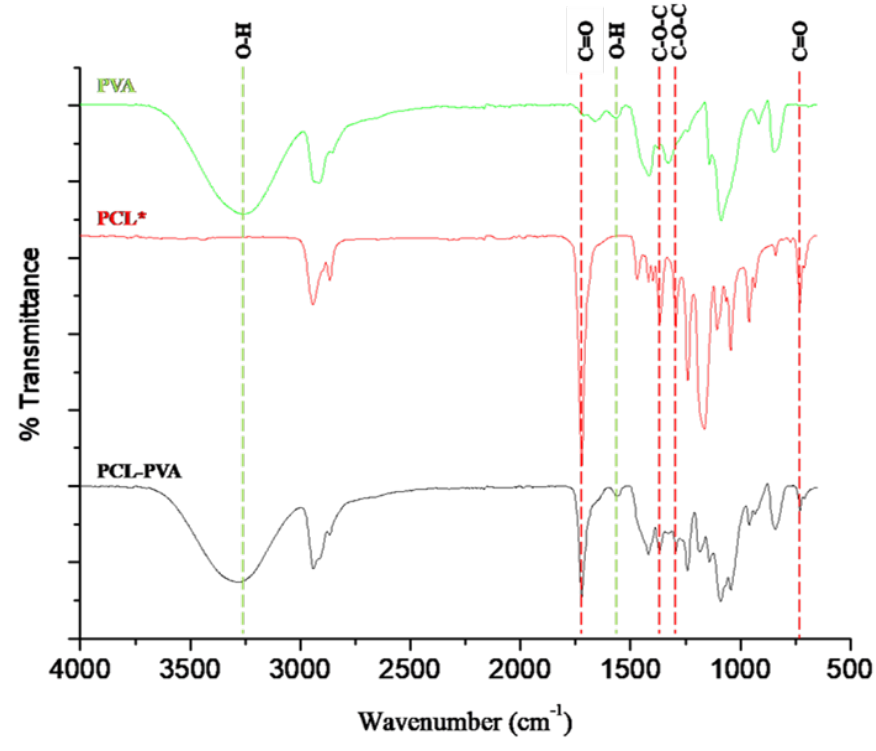

Fig. 5 ATR-FTIR spectra of PVA film, PCL film, and PVA-PCl hybridized film.

The enhancement of PVA-PCL hybridized film can also be proven by the study of contact angle. The hybridized film showed a contact angle of $75.9^{\circ}$, because of the presence of $\mathrm{OH}$ - bond from PVA. The PCL* film exhibited the contact angle of $82.7^{\circ}$, while PVA illustrated the contact angle of $61.3^{\circ}$. It is interesting to notice that the PCL* film prepared using a glacial acetic acid showed a relatively low contact angle, but the absence of $\mathrm{OH}$ - bond cannot enhance its water interaction and assist the polymer for better cell attachment and proliferation. The lack of interaction between water the PCL* film was also investigated in Fig. 7. When the PVA-PCL hybridized film and PCL* film were immersed in water at room temperature for two days, the hybridized film absorbed water and sink, but PCL* film did not absorb water and float. The water absorption of film is vital for scaffold application because an ideal scaffold must be able to absorb excess execute and maintain moisture condition during wound healing process.
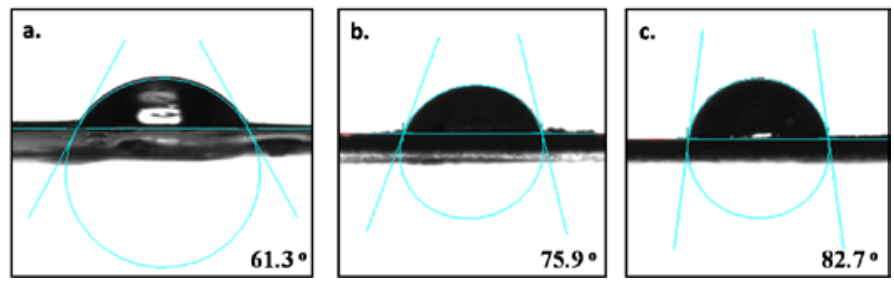

Fig. 6 Contact angle of (a) PVA film, (b) PVA-PCL hybridized film and (c) $P C L *$ film.

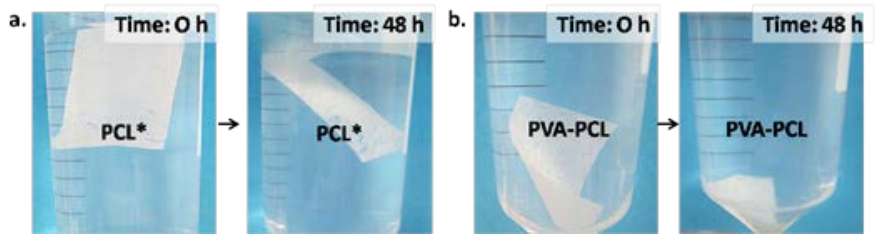

Fig. 7 The interaction between water and (a) PCL* film and (b) PVAPCL hybridized film.

Tensile strength is another significant factor that must taken into consideration during scaffold formation. Fig. 8 indicates the tensile strength of PVA film, PCL film, and PVA-PCL hybridized film in dry condition. Although the PVA film showed a very high tensile strength at 16.6 MPa in dry state, the film tends to fragile easily when it contacted water (Tomlinson and Carnali, 2007). As a matter of fact, this is a very worrying issue encountered by most of the hydrophilic polymeric scaffold. Because of the less stability in aqueous system, their beneficial function that improve cell attachment and proliferation 
was affected. The combination of of PVA and PCL into a hybridized scaffold resulting in a film with a relatively low tensile strength, i.e.7.4 MPa, which can remain relatively stable and less fragile when it comes into contact with water (Fig. 7 (a)). Besides, the hybridized film achieved a required tensile strength of skin scaffold that falling between 4.60 to $20 \mathrm{MPa}$. Although the tensile strength of PCL is low, about $2.4 \mathrm{MPa}$, it is very stable and not fragile when in contact with water (Fig. 7 (b)).

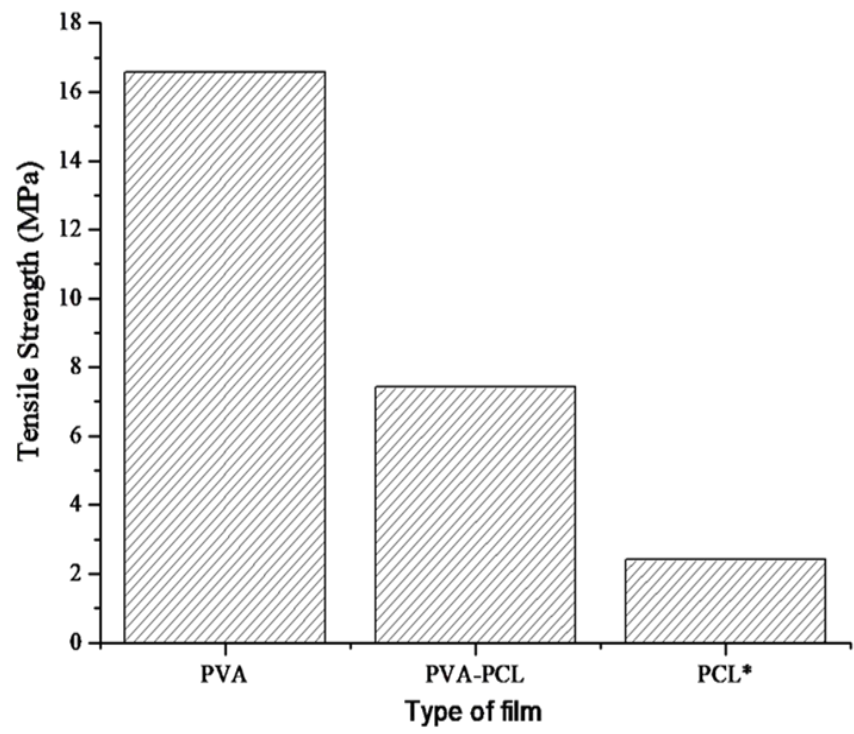

Fig.8 Tensile strength of PVA film, PVA-PCL hybridized film, and PCL* film in dry condition.

\section{CONCLUSION}

This investigation creates a breakthrough of combining a hydrophobic polymer, i.e. PCL, with a hydrophilic polymer, i.e. PVA, for biomedical applications because majority of hydrophilic polymer dissolved in this organic acid in certain concentration. A new binary solvent system containing glacial acetic acid and water with ratio 1:1 can be a proper solvent system used for preparing PCL (10 kDa)-PVA solution. The fabrication of PVA-PCL hybridized scaffold through casting can combine PVA and PCL into a scaffold without losing any important chemical properties of PVA and PCL. The new PVA-PCL hybridized scaffold has a moderate hydrophilic property, capable of absorbing water, and relatively strong at dry state. This finding is paramount of because it provides a simple and inexpensive way to prepare a PCL-PVA hybridized scaffold through less harmful solvent system for potential skin scaffold application.

\section{ACKNOWLEDGEMENT}

The authors would like to acknowledge Universiti Teknologi Malaysia (UTM) for providing the Research University Grant, Q.J130000.2509.20H51 as financial support of this project. The first author also wishes to thank the UTM for Zamalah scholarship.

\section{REFERENCES}

Access, O., Lepry, W. C., Smith, S., Liverani, L., Boccaccini, A. R., Nazhat, S. N. 2016. Acellular bioactivity of sol-gel derived borate glasspolycaprolactone electrospun scaffolds. Biomedical Glasses, 88-98.

Bloemen, M. C., Wal, M. B., Verhaegen, P. D., Nieuwenhuis, M. K., Baar, M. E., Zaijlen, P. P., Middelkoop, E. 2012. Clinical effectiveness of dermal substitution in burns by tropical negative pressure: A multicenter randomized controlled trial. Wound Repair Regeneration 20(6), 797-805.

Brigham, P. A., McLoughlin, E. 1996. Burn incidence and medical care use in the United Stated: estimates trends, and data sources. Journal of Burn Care Rehabilitation 17, 95-107.

Choi, E. G., Agrawal, A. 2010. A randomized, prospective, controlled study of forearm donor site healing when using a vacuum dressing. Otolaryngology-Head and Neck Surgery 142, 174-178.

Contardi, M., Heredia-Guerrero, J. A., Perotto, G., Valentini, P., Pompa, P. P., Spanò, R., Bayer, I. S. 2017. Transparent ciprofloxacin-povidone antibiotic films and nanofiber mats as potential skin and wound care dressings. European Journal of Pharmaceutical Sciences 104, 133-144.

Cossio, M. L. T., Giesen, L. F., Araya, G., Pérez-Cotapos, M. L. S., Vergara, R. L., Manca, M., Héritier, F. 2012. An alternative solvent system for the steady state electrospinning of polycaprolactone. Uma Ética Para Quantos XXXIII(2), 81-87.

Ferreira, J. L., Gomes, S., Henriques, C., Borges, J. P., Silva, J. C. 2014. Electrospinning polycaprolactone dissolved in glacial acetic acid: Fiber production, nonwoven characterization, and in Vitro evaluation. Journal of Applied Polymer Science 131(22), 37-39.

Hiep, N. T., Lee, B. T. 2010. Electro-spinning of PLGA/PCL blends for tissue engineering and their biocompatibility. Journal of Materials Science: Materials in Medicine 21(6), 1969-1978.

Kumbar, S. G., Nukavarapu, S. P., James, R., Nair, L. S., Laurencin, C. T 2008. Electrospun poly(lactic acid-co-glycolic acid) scaffolds for skin tissue engineering. Biomaterials 29, 4100-4107.

Liu, Y., Wang, S., Lan, W., Qin, W. 2017. Fabrication and testing of PVA chitosan bilayer. Coatings (7), 1-16.

Park, J. C., Ito, T., Kim, K. O., Kim, K. W., Kim, B. S., Khil, M. S., et al. 2010. Electrospun poly(vinyl alcohol) nanofibers: Effects of degree of hydrolysis and enhanced water stability. Polymer Journal 42, 273-276.

Santos, C., Silva, C. J., Büttel, Z., Guimarães, R., Pereira, S. B., Tamagnini, P. Zille, A. 2014. Preparation and characterization of polysaccharides / PVA blend nanofibrous membranes by electrospinning method. Carbohydrate Polymers 584-592.

Shalumon, K. T., Binulal, N. S., Selvamurugan, N., Nair, S. V., Menon, D., Furuike, T., et al. 2009. Electrospinning of carboxymethyl chitin/poly(vinyl alcohol) nanofibrous scaffolds for tissue engineering applications. Carbohydrate Polymers 77(4), 863-869.

Shao, C., Kim, H. Y., Ding, B. Lee, D. R., Park, S. J. (2003). Fiber mats of poly(vinyl alcohol)/silica composite via electrospinning. Materials Letters 57, 1579- 1584 .

Tomlinson, A. and Carnali, J. (2007). A review of key ingredients used in past and present autodishwashing formulations and the physic-chemical processes they facilitate. Handbook for cleaning/decontamination of surface 1

Ulrich, M. M. W. 2014 Regenerative medicine in burn wound healing: Aiming for the perfect skin. EWMA Journal 14(1), 25-28.

Wang, K. 2012 Small-diameter tissue engineered vascular graft made of electrospun PCL/lecithin blend. Colloids and Surfaces B: Biointerfaces 85(1) 32-39.

Woodruff, M. A., Hutmacher, D. W. (2010). The return of a forgotten polymer-polycaprolactone in the 21st century. Progress in polymer science 35(10), 1217-1256.

Yoshimotoa, H., Shina, Y. M., Teraia, H., Vacantia, J. P. 2003. A biodegradable nanofiber scaffold by electrospinning and its potential for bone tissue engineering. Biomaterials 24, 2077-2082.

Zhang, C., Yuan, X., Wu, L., Han, Y. and Sheng, J. (2005). Study on morphology of electrospun poly(vinyl alcohol) mats. European Polymer Journal 41, 423-432. 